\title{
Uterus transplantation: current progress and future prospects
}

\author{
This article was published in the following Dove Press journal: \\ International Journal of Women's Health \\ 5 February 2016 \\ Number of times this article has been viewed
}

\section{Liza Johannesson Stina Järvholm}

Department of Obstetrics and Gynecology, Sahlgrenska Academy, University of Gothenburg, Sahlgrenska University Hospital, Gothenburg, Sweden
Correspondence: Liza Johannesson Department of Obstetrics and Gynecology, Sahlgrenska University Hospital, 4I3 45 Gothenburg, Sweden Email liza.johannesson@vgregion.se

\begin{abstract}
Even if reproductive medicine has been remarkably successful during the past few decades, with the introduction of in vitro fertilization in the late 1970s and intracytoplasmic sperm injection in the early 1990s, it has been repeatedly mocked by infertility due to an absolute uterine factor. No treatment has been available for the women suffering from an absent or dysfunctional uterus, in terms of carrying a pregnancy. Approximately one in 500 women suffer from absolute uterine infertility, and the option so far to become a mother has been to either adopt or utilize gestational surrogacy. As of today, a total of eleven cases of human uterus transplantations have been reported worldwide, conducted in three different countries. The results of these initial experimental cases far exceed what might be expected of a novel surgical method. Many more uterus transplantations are to be expected in the near future, as other research teams' preparations are being ready to be put into clinical practice. In this review, we summarize the current worldwide experience of uterus transplantation as a treatment of absolute uterine factor infertility and the future prospects of human uterus transplantation.
\end{abstract}

Keywords: uterus, transplantation, infertility

\section{Introduction}

Infertility due to a lack of anatomical uterus or functional uterus, ie, an inability of the uterus to carry a pregnancy, has eluded reproductive medicine for a long period of time. Uterine factor infertility is estimated to affect thousands of women worldwide and can be caused by either congenital Müllerian malformations, such as in the Mayer-Rokitansky-Küster-Hauser (MRKH) syndrome, or more commonly acquired as in the cases of women suffering from Asherman's syndrome, pregnancy interfering myomas, or hysterectomies. Since no successful treatment has been available for absolute uterine factor infertility, the options for these women to become mothers have been either to adopt or to go through with gestational surrogacy, a procedure that is currently banned in many countries.

During the late 1970s, 1980s, and 1990s, the successful introduction of in vitro fertilization and intracytoplasmic sperm injection enabled fertility in the majority of infertile couples. Little attention was subsequently paid to research in the field of uterus transplantation, even though women with uterine factor infertility represent a substantial portion and is effectively the largest nontreatable fraction of the remaining infertile population. The research field was rediscovered in the early 2000s and has since then been evolving rapidly.

Uterus transplantation models have since been developed in several animal species starting with rodents, ${ }^{1,2}$ large domestic species, ${ }^{3,4}$ and finally, nonhuman primates ${ }^{5}$ before entering the experimental clinical stage. Successful pregnancies have been described in different species such as mouse, ${ }^{1,6}$ rat, ${ }^{7,8}$ sheep, ${ }^{3,9}$ and nonhuman primates. ${ }^{10}$ 
In addition, stable uterine allografts have also been achieved in large animals. ${ }^{11}$

A transplantation of a uterus, unlike any other organ transplantation, involves no less than four parties - recipient, donor, partner of the recipient, and the possible future child. All of them are exposed to potential risks if the surgery has to be performed. Uterus transplantation is a complex procedure and is surrounded by not only medical and psychological implications but also ethical, moral, and cultural concerns and expectations.

In 2014, the report of the first live birth following human uterus transplantation was published, showing that uterine factor infertility, even when considered absolute, is now treatable. ${ }^{12}$ This first birth has later been followed by three more births proving the outcome of uterus transplantation in this early stage of clinical implementation to be astonishing. ${ }^{13}$ In this article, the progress and future prospects of uterus transplantation is addressed.

\section{Human uterus transplantation}

Worldwide, a total of eleven cases of human uterus transplantations have so far been reported, conducted in three different countries and cultural settings (Table 1). ${ }^{14-16}$

The first uterus transplantation was reported in $2002 .^{15}$ A Saudi Arabian team then performed transplantation in a 26-year-old woman who lost her uterus due to peripartal bleeding 6 years earlier following a cesarean section. The graft was from a 46-year-old, previously healthy, woman who underwent hysterectomy when removing her ovaries due to benign bilateral multiloculated ovarian cysts. In order to prolong the short retrieved uterine vessels of the graft, patches from the great saphenous veins of the recipient were used and anastomosed to the external iliac vessels. During the first 3 months, cyclic hormonal therapy with estrogen and progesterone was given and two withdrawal bleedings occurred promptly after cessation of the hormones (Table 1). Three months postoperatively, the uterus was found to be necrotic and was removed (Table 1). The authors espoused inadequate tissue support of the graft, leading to tension and thrombosis of the supplying vessels, as a reason for this outcome.

The second human uterus transplantation was reported in 2011. ${ }^{17}$ A 23-year-old woman with MRKH syndrome and a previous jejunum-derived vaginal reconstruction was transplanted with a uterus from a deceased 21-year-old donor. The internal iliac artery and vein of the donor were anastomosed in an end-to-side fashion to the external iliac artery and vein of the recipient. After 20 days, the recipient had her first menstruation (Table 1). Another two irregular menstrual cycles happened, and she was subsequently given cyclic hormonal therapy providing monthly withdrawal bleedings.

The first clinical series of uterus transplantation cases was performed in Sweden in 2012/2013. ${ }^{14}$ The trial involved nine transplantations with uteri from live donors. During the initial 4 months, two of the women had to undergo hysterectomy, in one case due to thrombosis of the uterine arteries and in another case due to a severe untreatable intrauterine infection. The remaining seven women started menstruating during the first 4-8 weeks post surgery, and the grafts remained viable with regular menstruations during the posttransplantation years (Table 1). ${ }^{18}$

\section{Donors}

Uterus transplantation is not restricted to either live or deceased organ donation. According to the World Health Organization Guiding Principle, organ donations from

Table I Summary of the human uterus transplantation cases $(n=I I)$ according to complications, donor type, and graft outcome

\begin{tabular}{|c|c|c|c|c|c|c|c|}
\hline Recipient & Diagnosis & Donor & $\begin{array}{l}\text { Graft } \\
\text { failure }\end{array}$ & $\begin{array}{l}\text { Highest grade } \\
\text { of complication }\end{array}$ & $\begin{array}{l}\text { Highest grade of } \\
\text { donor complication }\end{array}$ & $\begin{array}{l}\text { Postoperative } \\
\text { menstruation }\end{array}$ & Pregnancy \\
\hline I & Peripartal bleeding & Live & $Y$ & Grade IIIb & Grade IIIb & $Y$ & $\mathrm{~N}$ \\
\hline 2 & $\mathrm{MRKH}$ & Deceased & $\mathrm{N}$ & & & $Y$ & $Y$ \\
\hline 3 & Cervical cancer & Live & $\mathrm{N}$ & Grade II & & $Y$ & $Y$ \\
\hline 4 & MRKH & Live & $Y$ & Grade IIIb & Grade IIlb & $\mathrm{N}$ & $\mathrm{N}$ \\
\hline 5 & MRKH & Live & $\mathrm{N}$ & & & $Y$ & $Y$ \\
\hline 6 & MRKH & Live & $\mathrm{N}$ & & & $Y$ & $Y$ \\
\hline 7 & MRKH & Live & $\mathrm{N}$ & Grade II & & $Y$ & $Y$ \\
\hline 8 & MRKH & Live & $\mathrm{N}$ & & & $Y$ & $\mathrm{~N}$ \\
\hline 9 & MRKH & Live & $\mathrm{N}$ & Grade II & & $Y$ & $Y$ \\
\hline 10 & MRKH & Live & $\mathrm{N}$ & Grade II & & $Y$ & $Y$ \\
\hline 11 & MRKH & Live & $Y$ & Grade IIIb & & $\mathrm{N}$ & $\mathrm{N}$ \\
\hline
\end{tabular}

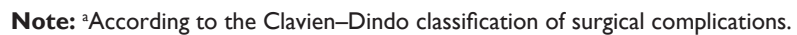

Abbreviations: Y, yes; N, no; MRKH, Mayer-Rokitansky-Küster-Hauser. 
deceased donors should always be developed to their maximum potential, evading the innate risks to live donors. ${ }^{19}$ However, because of the shortage of suitable organs from deceased donors, donations from live donor are necessary in order to meet current patient needs. Because of this, live donation is practiced despite the fact that it may involve potential risks for the donor that may not be negligible. In the eleven reported cases of human uterus transplantations, the uteri were from live donors in ten cases (Table 1) ${ }^{14,15}$ and from a deceased donor in one case. ${ }^{17}$ Both donor options carry their respective advantages and disadvantages.

\section{Live uterus donation}

When procuring a uterus from a live donor, it is possible to set the time for transplantation to a convenient date and the recipient can receive the transplant at a time when both parties are in an optimized and thoroughly prepared condition, thus increasing the odds of graft survival. This possibility to schedule the surgery also gives ample time to evaluate the donor and the organ prior to the transplantation. Exclusion of unsuitable donor candidates and organs of inferior quality is crucial to the outcome. The presence of systemic illness, donor infertility or subfertility, cervical or endometrial dysplasia, human papillomavirus infection, myomas, adenomyosis, polyps, vascular anatomy, and intrauterine adhesions should be thoroughly evaluated and considered before the procedure is carried out. ${ }^{20}$

Although the uterus is not a vital organ, the live uterus donor is still exposed to the risk of surgical complications during the retrieval. The first live uterus donor suffered from an intraoperative small laceration of the ureteral wall that was detected during the retrieval and sutured by an urologist perioperatively (Table 1). ${ }^{15}$ The third live donor (Case 2 in the Swedish trial) postoperatively developed an ureterovaginal fistula by day 16, and a pyelostomy catheter was inserted and the ureter reimplanted on day 134 (Table 1). ${ }^{14}$ The long durations of surgery for the live donors (mean, 12 hours ${ }^{14}$ ) pose an anesthesiological risk and increase complications correlated to extended time of surgery. The major time-consuming part of the procedure has been reported to be the difficult surgical isolation of the uterine vessels, in particular the veins. ${ }^{14}$ It has been suggested that a larger vein diameter like the ones of the ovarian veins would be preferable to use for anastomosis. ${ }^{21}$ This would require removal of the ovary itself, resulting in potential hormonal dysfunction in a premenopausal woman. In a live donor setting, the selection of the ovarian veins will only be possible in postmenopausal donors. The upper part of the uterine vessels, ie, the vessels connecting the uterine vessels to the ovarian vessels, may provide an adequately good substitute for anastomosis. With the development of techniques of uterus transplantation further, the risks of surgical complications are likely to decrease and new lessinvasive methods such as laparoscopic or robotic-assisted surgery may prove to be a useful tool for parts of the surgical retrieval procedure.

The psychological strain the live donor is exposed to is partly related to the outcome of the recipient. It has been shown in related living kidney donation that the well-being of the donor, particularly concerning sorrow and depressive symptoms, is affected by a poor recipient outcome of the transplantation. ${ }^{22,23}$ Nonetheless, since the uterus, unlike the kidney, is a nonvital organ, a uterine graft failure does not necessarily mean that the recipient is left in a bad medical condition as in other solid organ transplantations, which might warrant a reduced psychological strain on the donor.

\section{Deceased uterus donation}

The overshadowing benefit associated with deceased uterus donation is that there is no donor risk associated with surgery. Other potential benefits might be that the surgical dissection procedure is easier and takes a shorter time and that the vessels of larger diameter can be used for the anastomoses, also simplifying the transplantation procedure. In the Turkish uterus transplantation case, both the bilateral common iliac vessels and the ovarian vessels were included in the graft, providing the graft with vascular pedicles of ample length and thickness. ${ }^{16}$

In deceased donation, the ischemic time is generally longer than in live donation. A prolonged ischemic time is known to reduce graft function ${ }^{24}$ and increase the incidence of postoperative rejection, both acute and chronic. ${ }^{25}$ The tolerable ischemic time differs between organs and is not yet defined in a human uterus. ${ }^{26}$ There is no report of the total ischemic time in the Turkish case. ${ }^{16}$ However, the stated transfer time between the two different hospitals where the retrieval and the actual transplantation took place was only 30 minutes. Based on the histological laboratory findings, uterine myometrial and endometrial tissue can endure cold ischemia in Celsior solution for 24 hours and still show histological integrity. ${ }^{27}$ In another study, simulating multiorgan retrieval with concurrent uterus retrieval, no histological changes were found after 12 hours of cold ischemia in UW solution. ${ }^{28}$

From previous research in kidney transplantation, it is proven that the long-term graft viability is reduced when the graft is donated by a deceased donor. ${ }^{29}$ This might, at least 
partly, be explained by the brain death-induced systemic inflammation occurring in a deceased donor, which may have an adverse impact on the transplanted organ. ${ }^{30}$ Since the uterus is considered to be a temporary transplant and the long-term graft viability is of less importance, the influence of the systemic inflammation is likely not as central as in other solid organ transplantation settings. The possible scarcity of available uterine grafts from deceased donors might make it difficult to meet patient needs. The intended donor should not only meet all the donor criteria applied in a general organ transplantation setting but also be a female, preferably premenopausal; have a proven fertility; and not be previously hysterectomized, all of which may severely reduce the availability of suitable donors and organs.

\section{Uterus transplantation and immunology}

Since the induction of tolerance, precluding the need for maintenance immunosuppression, has proven to be elusive, immunosuppressive drugs are still used to minimize graft rejection, following organ transplantation. Finding the most favorable level of immunosuppression in solid organ transplantation is a balancing act between preventing rejection and the adverse effects of immunosuppressive drugs causing morbidity. The need for immunosuppressive medications is not constant, and the required initial high blood levels of immunosuppression can shortly be reduced to a lower maintenance blood level after transplantation. Induction therapy, ie, perioperative prophylactic immunosuppression, is commonly used to prevent acute rejection in the 1 st month after transplantation. The maintenance therapy is normally given as a combination of drugs with different pharmacokinetic mechanisms in order to minimize potential side effects.

In the first reported case of uterus transplantation in humans, the recipient was given induction therapy with corticosteroids both preoperatively (orally, $4 \mathrm{mg} / \mathrm{kg} / \mathrm{body}$ weight) and perioperatively (IV, $500 \mathrm{mg} \times 2$ ). Postoperatively, she was on a triple immunosuppression therapy with cyclosporine, azathioprine, and prednisolone.

The second uterus recipient ${ }^{16}$ was given induction with thymoglobulin $(2.0 \mathrm{mg} / \mathrm{dL}$ daily; days $0-10)$. Corticosteroids were given during surgery ( $1 \mathrm{~g}$ IV) and tapered during the 1 st postoperative week. As maintenance therapy, she was given tacrolimus $(0.2 \mathrm{mg} / \mathrm{kg})$ from day 7 onward (aiming for trough levels of 15-20 ng/mL), mycophenolate mofetil (MMF; $2 \mathrm{~g} / \mathrm{d}$ ), and corticosteroids (20 mg/d).

The nine Swedish recipients received induction with corticosteroids (IV, $500 \mathrm{mg}$ ) perioperatively and either thymoglobulin (IV, $2.5 \mathrm{mg} / \mathrm{kg}$ body weight) or antithymocyte globulin (IV, $5 \mathrm{mg} / \mathrm{kg}$ body weight) twice on the day of surgery. ${ }^{14}$ Maintenance therapy was with tacrolimus, aiming at trough levels of $10-15 \mathrm{ng} / \mathrm{mL}$ during the 1 st month and 5-10 ng/mL from the 2nd month onward. Oral MMF was given (1 g) preoperatively and from postoperative day 1 , administered twice daily, and the aim was to keep the MMF area under the curve trough levels at 40-60 mg/h/L. Six months post transplantation, the potentially teratogenic MMF was discontinued. In some cases, MMF had to be replaced with azathioprine.

\section{Rejection}

Following transplantation, the transplanted organ is at risk for rejection. The frequency of acute rejection episodes varies depending on which organ is transplanted. The highest incidence of acute rejection is shown after lung, heart, and intestinal transplantation $(-35 \%-40 \%, 30 \%-45 \%$, and $55 \%$, respectively). ${ }^{31-33}$ Liver and kidney transplantation generally shows less frequent episodes of acute rejection, with incidences of $13 \%-30 \%$ and $12 \%-14 \%$, respectively. ${ }^{34,35}$ The surveillance of organ functionality, particularly to detect the onset of rejection, is a crucial task for the long-term viability of the graft. Usually, diagnosis of acute rejection relies on clinical signs, but laboratory data such as blood markers (lipase/amylase in pancreas transplantation, creatinine in kidney transplantation, and liver enzymes in liver transplantation) are invaluable tools to monitor graft function. There is no specific blood marker for the uterus that reveals a decline in uterine function or rejection, and rejection might thus not be clinically detected until significant graft damage has occurred. As subclinical rejection episodes may occur, a noninvasive graft monitoring is desirable in all organ transplantation. These subclinical episodes of uterus rejection can only be detected with acute or protocol biopsies. The uterine graft is, unlike other solid organs, easily accessible from the vagina, and cervical tissue biopsies are, if not noninvasive, at least minimally invasive and provide an ample surveillance option of rejection. Unlike an endometrial biopsy, the cervical biopsy does not interfere with the cavity of the uterus and can therefore also function as surveillance of rejection during pregnancy.

In the Saudi Arabian case, the transplant was monitored by Doppler ultrasound, magnetic resonance imaging, and measurements of the CD4/CD8 ratio in peripheral blood. ${ }^{15}$ Nine days after surgery, the recipient expressed fatigue, malaise, and low abdominal and back pain. She showed subclinical fever, tachycardia, and a vaginal discharge predicted 
to be signs of acute rejection. She was initially treated with an increased temporary immunosuppression and IV corticosteroids, yet the episode of rejection was not resolved until antithymocyte globulin was given.

In the second case of uterus transplantation, the graft was monitored by biopsies from the transplanted vaginal rim every 2 weeks for the first 3 months. After 3 months, an endometrial biopsy was taken every 3 months. ${ }^{17}$ No reports of rejection episodes have been published.

In the Swedish trial, the recipients and their grafts were frequently examined clinically, including ultrasound evaluation of the endometrium and the uterus, Doppler ultrasound to evaluate the blood flow in the uterine arteries, and visual inspection of the cervix as well as cervical cultures and cervical biopsies. ${ }^{18}$ Occasional subclinical episodes of mild rejection were detected on cervical biopsies during the 1 st postoperative year, and the episodes were effectively reversed by short courses of increased immunosuppression. Rejection episodes were diagnosed based on the histopathological interpretation of the cervical biopsies and graded according to a grading system for uterine rejection that was originally developed for rejection in nonhuman primates without immunosuppression and later adopted by humans. ${ }^{11}$ The classification was done using a 4-grade scale running from no rejection to low-, medium-, and high-grade rejection (our unpublished data).

\section{Pregnancies and live births}

The major issues of uterus transplantation regarding immunosuppression and rejection can be summarized in three different areas of concern: the effect of pregnancy on graft rejection, the effect of the transplanted graft on pregnancy, and the effect of immunosuppression on both the fertility and the pregnancy outcome.

Throughout pregnancy, intake of immunosuppressive agents is vital to prevent organ rejection. All common medications used to avoid episodes of rejection cross the placenta barrier and subsequently reach the fetal circulation, thus exposing the child to potentially teratogenic agents during important developmental phases. ${ }^{36}$ Since the very first reported pregnancy following kidney transplantation with concurrent use of immunosuppression was reported in $1967,{ }^{37}$ data on children born to transplanted women have been reported, and the current data as of 2006 are exceeding 14,000 cases. ${ }^{38}$ Three large registers offer data about the outcome of pregnancies in transplant recipients, ${ }^{39-41}$ and all of these indicate similar trends of an increased incidence of obstetric complications, including ectopic pregnancy, hypertension and preeclampsia, miscarriage, premature delivery, low birth weight, stillbirth, and neonatal death. The potential adverse effects of immunosuppressive drugs are of a broad spectrum, ranging from severe malformations to delicate hardly detectable neurocognitive defects. The US Food and Drug Administration (FDA) has categorized immunosuppressive medications, and based on its recommendation, a ceased intake of some drugs is recommended prior to attempt of pregnancy. ${ }^{42}$ Using only the immunosuppressive drugs approved by the FDA during pregnancy, the risk of congenital malformations or anomalies in a pregnancy exposed to immunosuppression is comparable to the risk in a normal pregnancy.

During pregnancy, uterine and placental physiologic and hemodynamic changes occur, inducing changes in the plasma concentrations of drugs; hence, these need to be monitored thoroughly. ${ }^{43}$

Immunosuppressive doses often need to be increased during pregnancy and decreased in the postpartum period to achieve constant trough levels. Some studies report pregnant recipients requiring an almost twofold increase in doses compared with prepregnancy doses in order to keep trough level in a therapeutic window. ${ }^{44}$

Eighteen months after the second human uterus transplantation, the Turkish patient underwent two single embryotransfers and two pregnancies have been reported. ${ }^{45}$ The first pregnancy was biochemical, but the second was confirmed with visualization of an intrauterine gestational sac by ultrasound. This second pregnancy unfortunately ended in miscarriage prior to gestational week 8 .

The first human live birth was reported in $2014 .^{12}$ The recipient, a 35-year-old woman with MRKH syndrome, was transplanted with a live donor uterus from a 62-yearold postmenopausal family friend. Menstruation occurred 43 days post surgery and continued with regular intervals (26-36 days). During the first 12 months, she had two reversible episodes of mild rejection that was resolved with temporary IV corticosteroids. Twelve months after the transplantation, she had a single embryo-transfer that was immediately successful. During the pregnancy, the mother-to-be was on triple immunosuppression (tacrolimus, azathioprine, and corticosteroids), which continued up until delivery. One episode of mild rejection also appeared during the pregnancy (second trimester) but was reversed by IV corticosteroids. Fetal growth parameters and blood flows of the uterine arteries and umbilical cord were normal throughout pregnancy. Owing to preeclampsia, the patient was admitted in week $31+5$, and because of an abnormal CTG, a cesarean section 
was performed 15 hours after admission. A healthy boy baby of normal weight $(1,775 \mathrm{~g})$, in relation to gestational age, showing Apgar scores of 9, 9, and 10, was born. Examination of the placenta with the basal plate following cesarean section showed no changes affecting deep placentation. In the subsequent four pregnancies of the Swedish trial, the recipients showed occasional resolvable episodes of mild rejection and the children were born healthy (our unpublished data).

\section{Psychology}

Uterus transplantation raises questions not only of medical character but also of psychological aspects. Well-being among the participants is of great importance considering that uterus transplantation is a new type of a major experimental surgical procedure and infertility treatment.

In the two initial cases of human uterus transplantation, the psychology of the recipients or the live donor was not at all addressed. ${ }^{15,16}$ In the ongoing Swedish trial, the patient selection was based on physical inclusion criteria, ${ }^{14}$ but prior to inclusion of patients, a careful psychological evaluation was performed $^{46}$ according to a proposed psychological structure by the team (Table 2). A psychological protocol of this kind may lead to a number of patients being excluded. The main purpose should be to identify patients having inappropriate expectations of the procedure and thus might need more detailed psychological preparation or psychological support.

The ongoing Swedish trial aims to perform a continuing long-term psychological follow-up of the recipients and their partners and donors. ${ }^{46}$ Questionnaire data and recorded interviews from 2 years and 3 years after surgery are still to be reported (our unpublished data).

\section{Psychological evaluation of recipients and their partners}

In the Swedish trial, a licensed psychologist interviewed the couples well before the transplantation procedure (3-6 months) with the purpose to learn about mental strengths and vulnerability within each couple in a qualitative way. ${ }^{46}$ The interviews followed a semi-structured guide focusing on six main domains: psychological well-being, relationship, managing childlessness, knowledge about the project, and risk and relation with the donor. The recipients and their partners separately filled out standard questionnaires regarding mood, quality of life, relationship, and fertility

Table 2 Framework for psychological evaluation and follow-up

\begin{tabular}{|c|c|c|}
\hline Aims & Potential problems & Interventions \\
\hline \multicolumn{3}{|l|}{ Inclusion procedure } \\
\hline Selection of suitable and well- & Psychiatric burden & Questionnaires $^{\mathrm{a}}$ \\
\hline \multirow[t]{4}{*}{ informed individuals for the trial } & Donor issues & Semi-structured interviews \\
\hline & Unstable social situation & Establish contact for support regardless \\
\hline & Relationship burdens & of inclusion or exclusion \\
\hline & Medical issues & \\
\hline \multicolumn{3}{|l|}{ First 2 weeks } \\
\hline Medical/surgical stability & Adjustment disorder & Psychological support ${ }^{\mathrm{b}}$ \\
\hline Early return to function & High anxiety & Psychoeducation \\
\hline \multirow[t]{2}{*}{ Psychological stability } & Medical threats & \\
\hline & Relationship burdens & \\
\hline \multicolumn{3}{|l|}{ Up to 3 months } \\
\hline Graft rehabilitation & Relationship burdens & Psychological support ${ }^{\mathrm{b}}$ \\
\hline Reestablish intimacy with partner & Donor guilt & Questionnaires $^{\mathrm{a}}$ \\
\hline Return to everyday life & Depression/anxiety & \\
\hline Managing the waiting time prior to & Medical threats & \\
\hline embryo-transfer & Management of drug regimen & \\
\hline \multicolumn{3}{|l|}{ Up to 6 months } \\
\hline Managing the waiting time prior to & Relationship burdens & Psychological support ${ }^{\mathrm{b}}$ \\
\hline \multirow[t]{2}{*}{ embryo-transfer } & Depression/anxiety & Questionnaires $^{\mathrm{a}}$ \\
\hline & Medical threats & \\
\hline \multicolumn{3}{|l|}{ Up to I year } \\
\hline \multirow[t]{4}{*}{ Preparation for embryo-transfer } & Relationship burdens & Psychological support ${ }^{\mathrm{b}}$ \\
\hline & Depression/anxiety & Questionnaires $^{\mathrm{a}}$ \\
\hline & Medical threats & Semi-structured interviews \\
\hline & Concerns of pregnancy and parenthood & \\
\hline
\end{tabular}

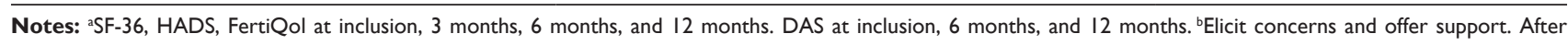
transplantation, adjustment to the new body function supporting going back to ordinary life. At I year, preparation for transition to the second phase of the trial (embryotransfer, pregnancy, and parenthood).

Abbreviations: SF-36, 36-item Short Form Health Survey; HADS, Hospital Anxiety and Depression Scale; FertiQoL, Fertility Quality of Life; DAS, Dyadic Adjustment Scale. 
quality of life (HADS [Hospital Anxiety and Depression Scale], PGWB [Psychological General Well-Being], DAS [Dyadic Adjustment Scale], SF-36 [36-item Short Form Health Survey]) (Table 2).

\section{Psychological evaluation of live donors}

The long-term psychosocial risk of live organ donors has so far not been widely explored. ${ }^{47}$ Data on the quality of life following other organ transplantation procedures such as live liver and kidney donation report that the quality of life for live donors was as good as or better than that of the general population or was comparable to a healthy control group. ${ }^{48-52}$ However, there are reports stating that depression, anxiety, and complicated family relations may be the result following organ donation. ${ }^{47,53-56}$ In the Swedish case series, ${ }^{57}$ the donors were evaluated preoperatively by a licensed psychologist, and to exclude potential bias and alliance with the parties, an independent psychologist did the evaluation of the couples. It was predicted that there would be a risk that possible strains of the donor or hesitation about donor suitability could be neglected in light of the wish to help the couple to proceed. The donors were also obliged to fill out the same questionnaires (except the form regarding fertility quality of life) as the recipients.

\section{Psychological outcome of recipients and their partners}

In the current Swedish trial, the recipients and their partners were psychologically stable and well prepared prior to transplantation. When the questionnaire scores were compared to the norm population, they were at inclusion as well or better off in general (Järvholm, Johannesson et al in press). They adjusted well to their new life situation, including mandatory medications and frequent and lengthy health care controls. The recipients with viable grafts expressed a significantly increased stress level 3 months after uterus transplantation and reported lower physical functioning and increased bodily pain. This strain regarding physical function after 3 months was also seen in the two women with graft failure, yet this group was too small in numbers to be statistically analyzed (Järvholm, Johannesson et al in press). Both recipients and partners had returned to baseline values at the 6-month follow-up. Regardless of the outcome of the transplant, it is important to recognize the impairment in quality of life of each individual and to offer support during these periods of need. In spite of personal outcome and adverse events, the recipients and their partners were found to be psychologically well adjusted 1 year after the transplantation. The recipients described the relationship with the donors as it had returned to "normal" or "better than before" even though some participants were mentally preoccupied with the well-being of the donor.

\section{Psychological outcome of live donors}

In the Swedish trial, the donors scored higher or equal to the general Swedish population at baseline regarding mood, well-being, relationship, and quality of life (HADS, PGWB, DAS, SF-36) (our unpublished results). However, high scoring at inclusion might also be due to the decisiveness and the motivation to suppress feelings of hesitation and the reluctances to report psychological strain from fear of being excluded as a donor. The qualitative evaluation is thus needed to address feelings of hesitation. Although the interviews revealed some mixed emotions, all donors were found to be stable and well adjusted for the trial, indicating that live uterus donors, despite their different previous experiences and the strains that in some cases occurred, tolerated the donation well, both medical and psychological. All donors returned to everyday life without major difficulties. None of the donors expressed regret about their decision to donate and would do it again if required.

\section{Future prospects}

The future of uterus transplantation is prone to hold modifications of the procedure. New methods to evaluate the recipients, donors, and organs, like angiographic mapping of vessels, preoperative or even perioperative, will possibly simplify the procedure and improve the outcome. There will certainly also be other surgical options, such as laparoscopic and robotic-assisted methods, giving the possibility to reduce the surgical duration and concurrent risks for both recipients and live donors. Extensive efforts are currently made in the area of bioengineered organs for transplantation purpose, the uterus not being an exception. The organ-engineering technology, being still in its infancy, pursues two ways of solution: the first involves donated organs, not suitable for transplantation, that is decellularized and the second alternative involves a synthetic matrix. The two different types of matrices would then after a recellularization process by the recipients own stem cells to be transplanted and in theory, function as good as any transplanted organ with the major benefit that no immunosuppression would be needed. At the time when uterus transplantation will enter the clinical arena in a wider perspective, the participants will express a broader diversity, both medically and psychologically. It will be of utmost importance to continue to develop and improve protocols for psychology with thorough assessment and support in a systematic and structured way. 


\section{Conclusion}

Uterus transplantation was a breakthrough in the field of reproductive medicine and has so far showed a remarkable successful outcome. Bearing this in mind, this procedure is still only proof of concept for uterus transplantation as a treatment for uterine factor infertility in a live related donor setting by laparotomic technique. Before introducing uterus transplantation in a wider general setting, several more carefully monitored pregnancies are required to evaluate major obstetrical risks, including miscarriage, preeclampsia, preterm birth, and fetal growth restriction. The concept of uterus transplantation will though surely be expanded to be demonstrated in other settings in the near future. All the current successful cases have been performed at a single institution, after years of meticulous research in several animal models. The years of extensive collaboration between gynecological and transplant surgeons, pathologists, and anesthesiologists is the single most important factor in achieving such a remarkable good outcome of this novel procedure. With more cases being performed in the near future, by new surgical teams and centers, one can expect a wider and more extensive variety of different complications and this might come to affect the overall outcome. Prior to the clinical introduction of uterus transplantation, it was debated whether it was ethically and morally defendable to perform the procedure. Now that it is proven to be successful in a controlled setting, the question might instead be whether it will be defendable or not to develop the uterus transplantation procedure further.

\section{Disclosure}

The authors report no conflicts of interest in this work.

\section{References}

1. Racho El-Akouri R, Wranning CA, Molne J, Kurlberg G, Brannstrom M. Pregnancy in transplanted mouse uterus after long-term cold ischaemic preservation. Hum Reprod. 2003;18(10):2024-2030.

2. Wranning CA, Akhi SN, Kurlberg G, Brannstrom M. Uterus transplantation in the rat: model development, surgical learning and morphological evaluation of healing. Acta Obstet Gynecol Scand. 2008; 87(11):1239-1247.

3. Dahm-Kahler P, Wranning C, Lundmark C, et al. Transplantation of the uterus in sheep: methodology and early reperfusion events. $J$ Obstet Gynaecol Res. 2008;34(5):784-793.

4. Wranning CA, El-Akouri RR, Lundmark C, et al. Auto-transplantation of the uterus in the domestic pig (Sus scrofa): surgical technique and early reperfusion events. J Obstet Gynaecol Res. 2006;32(4):358-367.

5. Johannesson L, Enskog A, Dahm-Kahler P, et al. Uterus transplantation in a non-human primate: long-term follow-up after autologous transplantation. Hum Reprod. 2012;27(6):1640-1648.

6. Racho El-Akouri R, Kurlberg G, Brannstrom M. Successful uterine transplantation in the mouse: pregnancy and post-natal development of offspring. Hum Reprod. 2003;18(10):2018-2023.

7. Wranning CA, Akhi SN, Diaz-Garcia C, Brannstrom M. Pregnancy after syngeneic uterus transplantation and spontaneous mating in the rat. Hum Reprod. 2011;26(3):553-558.
8. Diaz-Garcia C, Akhi SN, Wallin A, Pellicer A, Brannstrom M. First report on fertility after allogeneic uterus transplantation. Acta Obstet Gynecol Scand. 2010;89(11):1491-1494.

9. Wranning CA, Dahm-Kahler P, Molne J, Nilsson UA, Enskog A, Brannstrom M. Transplantation of the uterus in the sheep: oxidative stress and reperfusion injury after short-time cold storage. Fertil Steril. 2008;90(3):817-826.

10. Mihara M, Kisu I, Hara H, et al. Uterine autotransplantation in cynomolgus macaques: the first case of pregnancy and delivery. Hum Reprod. 2012; 27(8):2332-2340.

11. Johannesson L, Enskog A, Molne J, et al. Preclinical report on allogeneic uterus transplantation in non-human primates. Hum Reprod. 2013;28(1):189-198.

12. Brannstrom M, Johannesson L, Bokstrom $H$, et al. Livebirth after uterus transplantation. Lancet. 2015;385(9968):607-616.

13. Brannstrom M. The Swedish uterus transplantation project: the story behind the Swedish uterus transplantation project. Acta Obstet Gynecol Scand. 2015;94(7):675-679.

14. Brannstrom M, Johannesson L, Dahm-Kahler P, et al. First clinical uterus transplantation trial: a six-month report. Fertil Steril. 2014;101(5): $1228-1236$.

15. Fageeh W, Raffa H, Jabbad H, Marzouki A. Transplantation of the human uterus. Int J Gynaecol Obstet. 2002;76(3):245-251.

16. Ozkan O, Akar ME, Ozkan O, et al. Preliminary results of the first human uterus transplantation from a multiorgan donor. Fertil Steril. 2013; 99(2):470-476.

17. Ozkan O, Akar ME, Erdogan O, Ozkan O, Hadimioglu N. Uterus transplantation from a deceased donor. Fertil Steril. 2013;100(6):e41.

18. Johannesson L, Kvarnstrom N, Molne J, et al. Uterus transplantation trial: 1-year outcome. Fertil Steril. 2015;103(1):199-204.

19. Sixty-Third World Health Assembly, World Health Organization. WHO guiding principles on human cell, tissue and organ transplantation. Cell Tissue Bank. 2010;11(4):413-419.

20. Brannstrom M, Diaz-Garcia C, Hanafy A, Olausson M, Tzakis A. Uterus transplantation: animal research and human possibilities. Fertil Steril. 2012;97(6):1269-1276.

21. Kisu I, Banno K, Mihara M, et al. A surgical technique using the ovarian vein in non-human primate models of potential living-donor surgery of uterus transplantation. Acta Obstet Gynecol Scand. 2015; 94(9):942-948.

22. Ummel D, Achille M, Mekkelholt J. Donors and recipients of living kidney donation: a qualitative metasummary of their experiences. J Transplant. 2011;2011:626501.

23. Lentine KL, Schnitzler MA, Xiao H, et al. Depression diagnoses after living kidney donation: linking U.S. Registry data and administrative claims. Transplantation. 2012;94(1):77-83.

24. Quiroga I, McShane P, Koo DD, et al. Major effects of delayed graft function and cold ischaemia time on renal allograft survival. Nephrol Dial Transplant. 2006;21(6):1689-1696.

25. Schwarz A, Mengel M, Gwinner W, et al. Risk factors for chronic allograft nephropathy after renal transplantation: a protocol biopsy study. Kidney Int. 2005;67(1):341-348.

26. Stahl JE, Kreke JE, Malek FA, Schaefer AJ, Vacanti J. Consequences of cold-ischemia time on primary nonfunction and patient and graft survival in liver transplantation: a meta-analysis. PLoS One. 2008; 3(6): 2468 .

27. Sieunarine K, Lindsay I, Ungar L, Del Priore G, Smith JR. Cold ischaemic preservation of human uterine tissue. Int Surg. 2008;93(6): 366-372.

28. Del Priore G, Stega J, Sieunarine K, Ungar L, Smith JR. Human uterus retrieval from a multi-organ donor. Obstet Gynecol. 2007;109(1): 101-104.

29. Segev DL, Gentry SE, Warren DS, Reeb B, Montgomery RA. Kidney paired donation and optimizing the use of live donor organs. JAMA. 2005;293(15):1883-1890.

30. Pratschke J, Wilhelm MJ, Kusaka M, et al. Brain death and its influence on donor organ quality and outcome after transplantation. Transplantation. 1999;67(3):343-348. 
31. Smith JM, Skeans MA, Horslen SP, et al. OPTN/SRTR 2013 annual data report: intestine. Am J Transplant. 2015;15(suppl 2):1-16.

32. Valapour M, Skeans MA, Heubner BM, et al. OPTN/SRTR 2013 annual data report: lung. Am J Transplant. 2015;15(suppl 2):1-28.

33. Colvin-Adams M, Smith JM, Heubner BM, et al. OPTN/SRTR 2013 annual data report: heart. Am J Transplant. 2015;15(suppl 2):1-28.

34. Matas AJ, Smith JM, Skeans MA, et al. OPTN/SRTR 2013 annual data report: kidney. Am J Transplant. 2015;15(suppl 2):1-34.

35. Kim WR, Lake JR, Smith JM, et al. OPTN/SRTR 2013 annual data report: liver. Am J Transplant. 2015;15(suppl 2):1-28.

36. Ostensen M. Disease specific problems related to drug therapy in pregnancy. Lupus. 2004;13(9):746-750.

37. Board JA, Lee HM, Draper DA, Hume DM. Pregnancy following kidney homotransplantation from a non-twin. Report of a case with concurrent administration of azathioprine and prednisone. Obstet Gynecol. 1967;29(3):318-323.

38. Armenti VT, Daller JA, Constantinescu S, et al. Report from the National Transplantation Pregnancy Registry: outcomes of pregnancy after transplantation. Clin Transpl. 2006:57-70.

39. Armenti VT, Radomski JS, Moritz MJ, et al. Report from the National Transplantation Pregnancy Registry (NTPR): outcomes of pregnancy after transplantation. Clin Transpl. 2004:103-114.

40. Briggs JD, Jager K. The first year of the new ERA-EDTA Registry. Nephrol Dial Transplant. 2001;16(6):1130-1131.

41. Sibanda N, Briggs JD, Davison JM, Johnson RJ, Rudge CJ. Pregnancy after organ transplantation: a report from the UK Transplant pregnancy registry. Transplantation. 2007;83(10):1301-1307.

42. McKay DB, Josephson MA. Pregnancy in recipients of solid organs - effects on mother and child. N Engl J Med. 2006;354(12): 1281-1293.

43. Tendron A, Gouyon JB, Decramer S. In utero exposure to immunosuppressive drugs: experimental and clinical studies. Pediatr Nephrol. 2002; 17(2):121-130.

44. Akturk S, Celebi ZK, Erdogmus S, et al. Pregnancy after kidney transplantation: outcomes, tacrolimus doses, and trough levels. Transplant Proc. 2015;47(5):1442-1444.

45. Erman Akar M, Ozkan O, Aydinuraz B, Dirican K, Cincik M, Mendilcioglu I. Clinical pregnancy after uterus transplantation. Fertil Steril. 2013;100(5):1358-1363.
46. Jarvholm S, Johannesson L, Brannstrom M. Psychological aspects in pre-transplantation assessments of patients prior to entering the first uterus transplantation trial. Acta Obstet Gynecol Scand. 2015; 94(10):1035-1038.

47. Dew MA, Jacobs CL. Psychosocial and socioeconomic issues facing the living kidney donor. Adv Chronic Kidney Dis. 2012;19(4):237-243.

48. Trotter JF, Talamantes M, McClure M, et al. Right hepatic lobe donation for living donor liver transplantation: impact on donor quality of life. Liver Transpl. 2001;7(6):485-493.

49. Walter M, Dammann G, Papachristou C, et al. Quality of life of living donors before and after living donor liver transplantation. Transplant Proc. 2003;35(8):2961-2963.

50. Schulz KH, Kroencke S, Beckmann M, et al. Mental and physical quality of life in actual living liver donors versus potential living liver donors: a prospective, controlled, multicenter study. Liver Transpl. 2009; 15(12):1676-1687.

51. Benzing C, Hau HM, Kurtz G, et al. Long-term health-related quality of life of living kidney donors: a single-center experience. Qual Life Res. 2015:24(12):2833-2842.

52. Gross CR, Messersmith EE, Hong BA, et al. Health-related quality of life in kidney donors from the last five decades: results from the RELIVE study. Am J Transplant. 2013;13(11):2924-2934.

53. Clemens KK, Thiessen-Philbrook H, Parikh CR, et al. Psychosocial health of living kidney donors: a systematic review. Am J Transplant. 2006;6(12):2965-2977.

54. Jowsey SG, Schneekloth TD. Psychosocial factors in living organ donation: clinical and ethical challenges. Transplant Rev. 2008;22(3): 192-195.

55. Parikh ND, Ladner D, Abecassis M, Butt Z. Quality of life for donors after living donor liver transplantation: a review of the literature. Liver Transplant. 2010;16(12):1352-1358.

56. Dew MA, Dimartini AF, Devito Dabbs AJ, et al. Preventive intervention for living donor psychosocial outcomes: feasibility and efficacy in a randomized controlled trial. Am J Transplant. 2013;13(10):2672-2684.

57. Hellstrom M, El-Akouri RR, Sihlbom C, et al. Towards the development of a bioengineered uterus: comparison of different protocols for rat uterus decellularization. Acta Biomater. 2014;10(12):5034-5042.
International Journal of Women's Health

\section{Publish your work in this journal}

The International Journal of Women's Health is an international, peerreviewed open-access journal publishing original research, reports, editorials, reviews and commentaries on all aspects of women's healthcare including gynecology, obstetrics, and breast cancer. The manuscript management system is completely online and includes

\section{Dovepress}

a very quick and fair peer-review system, which is all easy to use. Visit http://www.dovepress.com/testimonials.php to read real quotes from published authors. 